\title{
DESEMPENHO ESCOLAR EM CRIANÇAS COM EPILEPSIA BENIGNA DA INFÂNCIA COM PONTAS CENTROTEMPORAIS
}

\author{
Lineu Corrêa Fonsecaㄹ ${ }^{1}$, Glória M.A.S. Tedrus ${ }^{1}$, Josiane Marias de Freitas Tonelotto², \\ Thais de Assis Antunes ${ }^{3}$, Marcelo Gulini Chiodi
}

\begin{abstract}
RESUM 0 - Aspectos psicossociais em crianças com epilepsia benigna da infância com pontas centrotemporais (EBICT) são objeto de controvérsias. 0 objetivo desta pesquisa foi estudar o desempenho escolar em crianças com EBICT. Vinte crianças foram submetidas ao Teste de Desempenho Escolar (TDE) e comparadas a crianças sadias pareadas por idade e escolaridade. Foram estudadas as relações entre o TDE e a lateralidade do foco e o número de descargas ao eletrencefalograma. As crianças com EBICT tiveram, de modo significativo, mais freqüentemente do que as sadias, desempenho inferior no subteste de leitura e no escore total. As crianças com desempenho inferior em leitura apresentaram maior número de descargas do que aquelas com desempenho médio e superior. Não houve diferenças no TDE segundo a lateralidade do foco. 0 número de descargas, ao interferir com a função cerebral, pode ser um fator a explicar o desempenho mais baixo na leitura.
\end{abstract}

PALAVRAS-CHAVE: epilepsia idiopática, eletrencefalograma, atividade epileptiforme, leitura.

\begin{abstract}
School performance in children with benign childhood epilepsy with centrotemporal spikes
ABSTRACT - Neuropsychological implications of benign childhood epilepsy with centrotemporal spikesrolandic spikes (BECTS) have not been adequately investigated. The aim of this study was to compare the results in a school performance test of patients with BECTS and normal age-matched controls. A total of 20 children with BECTS and 20 normal controls were submitted to anamnesis, clinical evaluation, Raven test, school performance test (SPT), digital electroencephalogram and quantitative electroencephalogram analysis. Comparing with normal controls, children with BECTS showed significantly lower SPT results, especially in reading test. There was an association between the higher number of rolandic spikes and inferior performance in SPT reading test. These findings suggest that discharges may be a factor in the genesis of lower performance in reading test in children with BECTS.

KEY WORDS: rolandic epilepsy, electroencephalogram, epileptiform activity, reading, cognition.
\end{abstract}

A epilepsia benigna da infância com pontas centrotemporais (EBICT) é uma forma de epilepsia, a mais comum na infância, para a qual não existem lesões anatômicas demonstráveis e em que há remissão espontânea das crises. 0 diagnóstico desta síndrome epiléptica é feito face à associação da história de crises e eletrencefalograma (EEG) sugestivos, na ausência de déficit neurológico ou intelectual ${ }^{1-3}$. Vários autores assinalam que as crianças com EBICT não são diferentes das crianças normais em relação a inteligência, desempenho escolar, comportamento e desenvolvimento ${ }^{4}$. Por outro lado, várias pesquisas têm mostrado alterações em testes neuropsicológicos especiais. Piccirilli et al. ${ }^{5,6}$ encontraram comprometimento neuropsicológico discreto, sugerindo que as descargas pudessem interferir com o processo de lateralização da linguagem. D 'Alessandro et al. ${ }^{7}$ descreveram distúrbio no desempenho cognitivo, especialmente nas crianças com descargas no hemisfério cerebral esquerdo. A importância das descargas na gênese de distúrbios neuropsicológicos tem apoio no achado de distúrbios cognitivos transitórios durante as mesmas ${ }^{8}$.

$M$ ais recentemente, foram relatados déficits neuropsicológicos, intelectuais e do comportamento em crianças com descargas centrotemporais, com ou sem crises epilépticas ${ }^{9}$ e que essas alterações comportamentais podem melhorar quando há redução no número de atividade epileptiforme no EEG, com 0 tratamento ${ }^{10}$. No entanto, os estudos sobre a relação entre o número de descargas ao EEG e o desempenho em testes

\footnotetext{
1Professor Titular de Neurologia da Faculdade de Medicina da Pontifícia Universidade Católica de Campinas, Campinas SP, Brasil (PUC-Campinas); ${ }^{2}$ Professor da Faculdade de Psicologia da PUC-Campinas; ${ }^{3}$ Bolsista CNPq/PIBIC - Brasil.
} 
Tabela 1. Distribuição dos indivíduos do grupo EBICT e do grupo controle segundo o desempenho no Teste de Desempenho Escolar. Média do número de pontas por minuto ao EEG segundo os resultados no TDE.

\begin{tabular}{|c|c|c|c|}
\hline Resultados do TDE & $\begin{array}{c}\text { EBICT } \\
\text { № de casos }\end{array}$ & $\begin{array}{c}\text { Grupo controle } \\
\text { № de casos }\end{array}$ & $\begin{array}{c}\text { Média do no de } \\
\text { pontas por minuto }\end{array}$ \\
\hline \multicolumn{4}{|l|}{ Leitura $^{1}$} \\
\hline Superior ou médio & 5 & 15 & $5,0^{2}$ \\
\hline Inferior & 15 & 5 & $11,7^{2}$ \\
\hline \multicolumn{4}{|l|}{ Escrita $^{3}$} \\
\hline Superior ou médio & 10 & 15 & 8,1 \\
\hline Inferior & 10 & 5 & 11,7 \\
\hline \multicolumn{4}{|l|}{ Aritmética } \\
\hline Superior ou médio & 9 & 8 & 7,7 \\
\hline Inferior & 11 & 12 & 10,9 \\
\hline \multicolumn{4}{|l|}{ TDE Total ${ }^{4}$} \\
\hline Superior ou médio & 7 & 13 & 7,9 \\
\hline Inferior & 13 & 7 & 10,5 \\
\hline
\end{tabular}

apresentam resultados discordantes ${ }^{11,12}$. Uma questão de importância é saber se essas alterações em testes especiais chegam a interferir significativamente em aspectos da vida diária como, por exemplo, no desempenho escolar. Neste sentido, foi relatada maior freqüência de dificuldades escolares em crianças com EBICT ${ }^{13}$ assim como desempenho inferior em teste de desempenho escolar ${ }^{12}$.

Este estudo tem como objetivo aprofundar os conhecimentos sobre desempenho escolar em crianças com EBICT e sua relação com a lateralidade do foco e o número de descargas epileptiformes.

\section{MÉTODO}

Participaram deste estudo 20 crianças com idade entre 7 e 11 anos, com diagnóstico de Epilepsia Benigna da Infância com pontas centro temporais segundo a Classificação Internacional das epilepsias e síndromes epilépticas ${ }^{14}$ (Comission) e os critérios de Dalla Bernardina et al. ${ }^{1}$. Os sujeitos foram selecionados a partir dos ambulatórios de neurologia e do serviço de eletrencefalografia do Hospital e Maternidade Celso Pierro PUC-Campinas.

A última crise epiléptica ocorreu há mais de 15 dias das avaliações. Treze crianças faziam uso de antiepilépticos e 7 estavam sem medicação.

Foi constituído um grupo controle de 20 crianças pareadas quanto a idade e escolaridade materna e que não apresentavam antecedentes sugestivos de patologias com envolvimento do sistema nervoso central (SNC), alterações ao exame neurológico ou ao EEG.
A inclusão no estudo somente foi realizada após o consentimento dos pais e esclarecidas as informações a respeito do tipo de estudo e procedimentos, conforme parecer do Comitê de Ética em Pesquisa em Seres Humanos da PUC-Campinas.

Foi aplicado o Teste de Desempenho Escolar (TDE) ${ }^{15}$ - instrumento psicométrico, desenvolvido e padronizado no Brasil (Porto Alegre- RS), que busca oferecer de forma objetiva uma avaliação das capacidades fundamentais para o desempenho escolar, mais especificamente da escrita, aritmética e leitura. Neste teste, o desempenho é classificado nas categorias superior, médio e inferior.

0 EEG digital (Braintech 3.0 - EMSA Equipamentos Médicos) foi realizado durante vigília, em repouso, durante hiperventilação e quando possível durante sono. Os eletrodos foram colocados segundo 0 sistema internacional $10-20^{16}$ com os eletrodos auriculares como a referência.

Foram contadas as descargas epileptiformes de localização central e/ou temporal média durante 5 minutos, em sequêencia, na maioria dos casos durante vigília e, então, calculada a média por minuto.

Foi avaliada a lateralização do foco ao EEG levando em conta a ocorrência unilateral das descargas ou seu franco predomínio em um dos hemisférios cerebrais.

Foram utilizados testes estatísticos não paramétricos nas situaç̃ões pertinentes. 0 nível de significância foi 0,05 .

\section{RESULTADOS}

As crianças com EBICT tiveram, de modo significativo (prova exata de Fisher, $p<0,05$ ), mais freqüentemente do que 
as sadias, desempenho inferior no subteste de leitura. Nos subtestes de escrita e no escore total houve tendência à ocorrência de desempenho inferior com maior freqüência nas crianças com EBICT $(p<0,010)$, enquanto que, em aritmética, não houve diferenças significativas (Tabela 1 ).

0 uso de medicação antiepiléptica não interferiu no desempenho no TDE.

Em relação à lateralidade do foco, não foram encontradas diferenças estatisticamente significativas $(p=0.39)$ entre a localização do foco e o desempenho escolar das crianças no TDE.

Quanto ao número de descargas ao EEG (Tabela 1), as crianças com desempenho inferior nos testes do TDE tiveram maior número de descargas no EEG do que aquelas com desempenho médio e superior, mas a diferença foi estatisticamente significativa apenas para 0 subteste de leitura ( $p=0,01$, prova de Kruskal-Wallis). Não foram observadas relações estatisticamente significativas entre a quantidade de descargas epileptiformes e o desempenho escolar dos sujeitos nos testes de escrita e aritmética do TDE.

\section{DISCUSSÃO}

Dalla Bernardina et al. ${ }^{17}$ apontam que a ausência de déficit neurológico e intelectual faz parte da definição da EBICT. No entanto, Deonna ${ }^{13}$ observou a maior freqüência de dificuldades escolares e neuropsicológicas em crianças pertencentes a este grupo. Desempenho inferior no Teste de Desempenho Escolar global havia sido relatado por M iziara ${ }^{12}$ que, no entanto, não referiu os achados em subtestes.

No presente estudo, as crianças com EBICT obtiveram resultados inferiores mais freqüentes do que as crianças sadias, especialmente, no subteste de leitura. Foi também observado que as crianças com desempenho inferior no teste de leitura tinham, de modo significativo, maior número de descargas ao EEG o que sugere a influência que as descargas têm sobre esta função cognitiva. Quanto à lateralidade do foco, uma das limitações do nosso estudo é o número pequeno de casos.

0 comprometimento da linguagem e da leitura na EBICT pode ser esperado devido à sobreposição das áreas corticais da linguagem com a atividade epiléptica. As descargas tendem a ser concentrar sobre a região central com uma predominância na porção interior da área rolandica e região silviana, às vezes se estendendo para a região temporopariental adjacente. Estudos utilizando PET (Positron Emission Tomography) têm demostrado que 0 córtex perissilviano deve ser 0 centro do processamento da linguagem e consequentemente da leitura ${ }^{18}$.

Binnie ${ }^{19}$ sugeriu que a presença de descargas epilépticas seria suficiente para provocar disfunções cognitivas tais como memória e linguagem, independentemente da ocorrência ou não de crises epilépticas.
Comprometimento na leitura e no aprendizado auditivoverbal já haviam sido apontados em crianças com EBICT ${ }^{11}$. Carlsson et al. ${ }^{20}$, em estudo comparativo entre pacientes com dislexia com e sem descargas epilépticas na região rolândica ao EEG e grupo controle normal, concluíram que o grupo de portadores de anormalidades eletrencefalográficas apresentou maiores erros durante a leitura e maior comprometimento da atenção auditiva, mesmo após o desaparecimento das descargas epilépticas, quando comparado aos dois grupos sem descargas.

Embora a EBICT tenha vários aspectos benignos, como 0 desaparecimento espontâneo das crises e a não existência de lesões cerebrais, algumas crianças com essa síndrome podem ter repercussões neuropsicológicas de certa relevância. Um dos possíveis mecanismos é o comprometimento funcional desencadeado pelas descargas.

\section{REFERÊNCIAS}

1. Dallla Bernadina B, Chiamenti C, Capovilla G, Colamaria V. Benign partial epilepsies in childhood. In Epileptic Syndromes in Infancy, chilhood and adolescense. London: John Libey Eurotext, 1985:137-149.

2. Fonseca LC, Tedrus GMA. Epilepsia com pontas centrotemporais e com pontas parietais: estudo comparativo. Arq Neuropsiquiatr 1995;53: 208-212.

3. Miziara CSMG, Manreza MLG. Benign focal epilepsy of childhood with centrotemporal spikes (BECTS): clinical characteristics of seizures according to age at first seizure. Arq Neuropsiquiatr 2002;60:390-394.

4. Panayiotopoulos CP. Benign childhood partial seizures and related epileptic syndromes. London: John Libbey, 1999:33-70.

5. Piccirilli M, D'Alessandro P, Ticci C, Ferroni A . Language lateralization in children with benign partial epilepsy. Epilepsia 1988;29:19-25.

6. Piccirilli M, D'alessandro P, Sciarma, TE. Attention problem in epilepsy: possible significance of the epileptogenic focus. Epilepsia 1994;35:1091-1096.

7. D`Alessandro M, Piccirilli M, Tiacci C, et al. Neuropsychological features of benign partial epilepsy in children. It J Neurol Sci 1990;11:265-269.

8. Binnie CD, Silva M, Hurst A. Rolandic spikes and cognitive function Epilepsy Res 1992;6:71-73.

9. Weglage J, Demsky A, Pietsch M, Kurlemann G. Neuropsychological, intellectual and behavioral findings in patients with centrotemporal spikes with or without seizures. Dev Med Child Neurol 1997;39:646-651.

10. Pressler R, Robinson RO, Wilson GA, Binnie CD. Treatment of interictal EEG discharges improves behaviour in children with epilepsy. Epilepsia 2002;43(Suppl):8.

11. Staden U, Isaaca E, Boyd SG. Language dysfunction in children with rolandic epilepsy. Neuropediatrics 1998;29:242-248.

12. Miziara CSMG. Avaliação das funções cognitivas na epilepsia focal benigna da infância com descargas centrotemporais. Tese de doutorado. Universidade de São Paulo. São Paulo, 2003.

13. Deonna T. Rolandic epilepsy: neuropsychology of the active epilepsy phase. Epileptic Disorders 2000;2:(Suppl):59-61.

14. Commission on Classification and Teminology of the International League against Epilepsy. Proposal for revised classification of epilepsies and epileptic syndromes. Epilepsia 1989;30:389-399.

15. Stein LM. Teste de desempenho escolar: manual para aplicação e interpretação. São Paulo: Casa do Psicólogo Livraria e Editora, 1994.

16. Jasper HH. The ten-twenty system of the International Federation. Electroencephalogr Clin Neurophysiol 1958;10:371-373.

17. Dalla Bernardina B, Sgro V, Fejerman N. Epilepsy with centro-temporal spikes and related syndromes. In Roger J, Bureau M, Dravet C, Genton $\mathrm{P}$, Tassinari C A, Wolf P (eds). Epileptic syndromes in infancy, childhood and adolescense. London: John Libbey, 2002:181-202.

18. Stowe LA, Albertus WA, Willemsen A, Reuland E, Paans MJ, Vaalburg W. PET studies of language: an assessment of the reliability of technique. J Psycholo Res 1994;23:499-527.

19. Binnie CD. Significance and management of transitory cognitive impairment due to subclinical EEG discharge in children. Brain Dev 
1993;5:23-30.

20. Carlsson G, Igelbrink-Schulze N, Neubauer BA, Stephani U. Neuropsychological long-tem outcome of rolandic EEG traits. Epileptic Disorders 2000;2:(Suppl):63-66. 